\title{
(1) BIOENG
}

\section{ANÁLISE MULTICRITÉRIO PARA AVALIAÇ̃̃o DE CARTEIRAS DE PROJETOS APLICADA AOS PROJETOS DE PESQUISA E DESENVOLVIMENTO DO BIOEN LIGADOS À ETAPA AGRÍCOLA}

\author{
D. S. F. Lamarca*; M. M. Magalhães; S. S. Braga Junior
}

UNESP - Univ. Estadual Paulista, Campus de Tupã, SP, Brasil

Article history: Received 04 December 2015; Received in revised form 22 February 2016; Accepted 24 February 2016; Available online 21 March 2016.

\section{RESUMO}

Este estudo tem como finalidade prover insumos para a avaliação do desempenho de carteiras de projetos de P\&D do Programa de Pesquisa em Bioenergia (BIOEN), da Fundação de Amparo à Pesquisa do Estado de São Paulo (FAPESP), utilizando métodos de pesquisa de apoio aos processos de decisão multicritério em avaliação de projetos. É preciso uma combinação de metodologias para a avaliação de carteira de projetos por meio de adaptação de práticas já consagradas no mercado e pesquisas em termos de gestão de projetos de P\&D. Assim, realizou-se nesta pesquisa uma análise multicritério para organizar os projetos em carteiras (tipos de projetos) ligadas à etapa agrícola. Utilizando a metodologia de análise multicritério, foi enviado um questionário eletrônico para especialistas da área. Sendo assim, com as respostas coletadas, foram elaboradas cinco carteiras de projetos ligados à etapa agrícola do processo de produção de etanol de segunda geração. Como contribuição, as formações destas carteiras geram informação para processos de tomada de decisão no plano de negócios (investimentos), os quais podem ser utilizados aos produtos e processos do BIOEN.

Palavras-chave: Bioenergia; Carteira de projetos; Análise Hierárquica de Processos.

\section{MULTICRITERIA ANALYSIS FOR PROJECT PORTFOLIO EVALUATION APPLIED TO RESEARCH PROJECTS AND DEVELOPMENT BIOEN FOR STAGE AGRICULTURAL}

\begin{abstract}
This study aims to provide inputs for evaluating the performance of portfolios of projects of R \& D Research Program on Bioenergy (BIOEN), the Support Foundation of São Paulo (FAPESP), using methods of supporting research to multi-criteria decision-making processes in project evaluation. A combination of methodologies for the assessment of project portfolio through adaptation practices already established in the market and research in terms of $\mathrm{R} \& \mathrm{D}$ project management is necessary. Thus, this research took place one multi-criteria analysis to organize projects in portfolios (types of projects) related to the agricultural stage. Using the methodology of multi-criteria analysis, an electronic questionnaire was sent to experts in the field. Thus, with the collected responses were prepared five portfolios of projects related to agricultural second generation step of the ethanol production process. As a contribution, the formations of these portfolios generate information for decision-making
\end{abstract}

\footnotetext{
"lamarca@tupa.unesp.br
} 
processes in the business plan (investments), which can be used to BIOEN products and processes.

Keywords: Bioenergy; Project portfolio; Hierarchical analysis processes.

\section{INTRODUÇÃO}

Atualmente, o principal produto de substituição para a gasolina é o biocombustível etanol, sua importância vem desencadeando diversos estudos para melhorar sua produtividade no campo e também na indústria. As principais vantagens do etanol quando comparado a gasolina é o fato de ser fabricado por meio de fontes renováveis e liberar uma menor quantidade de gases poluidores no meio ambiente.

Lançado em 2008, o Programa de Pesquisa em Bioenergia (BIOEN) buscou estimular a pesquisa e articular o desenvolvimento utilizando laboratórios industriais e acadêmicos favorecendo assim, o progresso do conhecimento e a sua aplicação nos setores de bioenergia nacional. Além da pesquisa acadêmica, o BIOEN incentivou pesquisas cooperativas para o desenvolvimento de atividades entre universidades, empresas e institutos de pesquisa do Estado de São Paulo (FAPESP, 2014).

Desse modo, as linhas de pesquisa do programa seguem nas seguintes áreas: (I) Biomassa para Bioenergia (cana de açúcar em particular); (II) Processo de Fabricação de Biocombustíveis; (III) Bio-refinarias e Alcoolquímica, (IV) Aplicações de Etanol para Motores Automotivos (motores de combustão internas e células-combustível) e; (V) Pesquisa sobre Impactos Socioeconômicos, Ambientais e do Uso da Terra (FAPESP, 2014).

Considerando esta divisão de áreas, para a indústria de bioenergia, os impactos de um projeto estão além do seu desempenho, dependem também de sua influência sobre outros projetos de Pesquisa e Desenvolvimento (P\&D) desenvolvidos.

Este aspecto é reforçado por Mihm, Loch e Huchzermeier (2003) explicando que as decisões tomadas por outros pesquisadores podem influenciar o desempenho dentro de programas com diversas áreas, podendo gerar pressão sobre o cronograma e custos, tendo como consequência, a possibilidade do fracasso. Sendo assim, as avaliações individuais não são auto-suficientes, na qual torna-se necessário avaliar o impacto de carteiras de projetos de P\&D com o intuito de mensurar o desempenho geral do programa.

É no desempenho de um conjunto de projetos que se encontra o maior desafio da avaliação de um programa ou de uma carteira de projetos de P\&D, principalmente para o desempenho técnico e mercadológico. Em função disso, mensurar os impactos de projetos individuais pode ser uma tarefa demorada e complexa. Para solucionar esta questão, seria ideal considerar o nível adequado para uma análise dos impactos tecnológicos.

Selecionar os projetos de P\&D do Programa de Pesquisa em Bioenergia (BIOEN), para uma avaliação e posterior formulação em carteiras de projetos, agrupando-se os trabalhos ligados á etapa agrícola de produção, foi o problema da presente pesquisa.

Neste cenário, utilizou um modelo multicritério para avaliar projetos de $P \& D$ com o objetivo de construir carteiras de projetos com o intuito de analisar o desempenho de uma seleção restrita de trabalhos do Programa de Pesquisa em Bioenergia (BIOEN), da Fundação de Amparo à Pesquisa do estado de São Paulo (FAPESP), na qual estão ligados à etapa da fase agrícola do processo de produção de biocombustível.

As carteiras de projetos foram elaboradas e analisadas na análise dos resultados a partir da utilização de metodologias de análise hierárquica de 
processos, desenvolvendo informações para a tomada de decisões nos planos de negócios, dos quais se pode aplicar aos processos e produtos do BIOEN.

Desse modo, é importante destacar que a análise deste artigo está voltada apenas para os projetos relacionados com a

\section{REVISÃO BIBLIOGRÁFICA}

\subsection{Setor Sucroenergético}

Trata-se de um setor com grande mercado consumidor mundial e ligado diretamente as questões ambientais por emitir em sua queima menos poluentes em relação aos combustíveis fósseis. Esta questão evidência um panorama de demanda futura de produção de etanol com um cenário de ascensão que pode se restringir quanto a sua expansão por um quadro de crise econômica que acaba sendo um obstáculo para o avanço do setor.

O Brasil dentro do contexto mundial segue em segundo lugar com a fabricação de 6,2 bilhões de galões de etanol, sendo responsável por aproximadamente $25 \%$ da produção mundial, ficando atrás somente dos Estados Unidos, no qual representa $60 \%$ da produção mundial de etanol (RENEWABLE FUELS ASSOCIATION, 2015). etapa agrícola dentro do BIOEN, pois para realizar uma avaliação do sistema em geral, seria necessário um estudo mais aprofundado sobre o assunto, abrangendo outros temas como, por exemplo, a etapa industrial.

Diante deste contexto, para analisar os pontos em que estão inseridos os projetos BIOEN dentro da cadeia de suprimento, precisa-se analisar o desempenho da cadeia de suprimento deste produto. Desse modo, a cadeia de suprimento caracteriza o processo de gestão, no qual, existe o fornecimento de produtos, serviços e informações a partir do fornecedor primário, com o objetivo de agregar valor ao cliente. Tal modelo de gestão envolve a integração da operação dos negócios perpassando as fronteiras da logística (COOPER \& ELLRAM, 1993).

A cadeia sucroenergética está subdividida nas seguintes etapas: Fase Campo, Logística, Fase Industrial e Distribuição. A seguir, a Figura 1 ilustra todos os elos para a produção do etanol de primeira geração. 
Fluxograma de produção de álcool e açúcar.

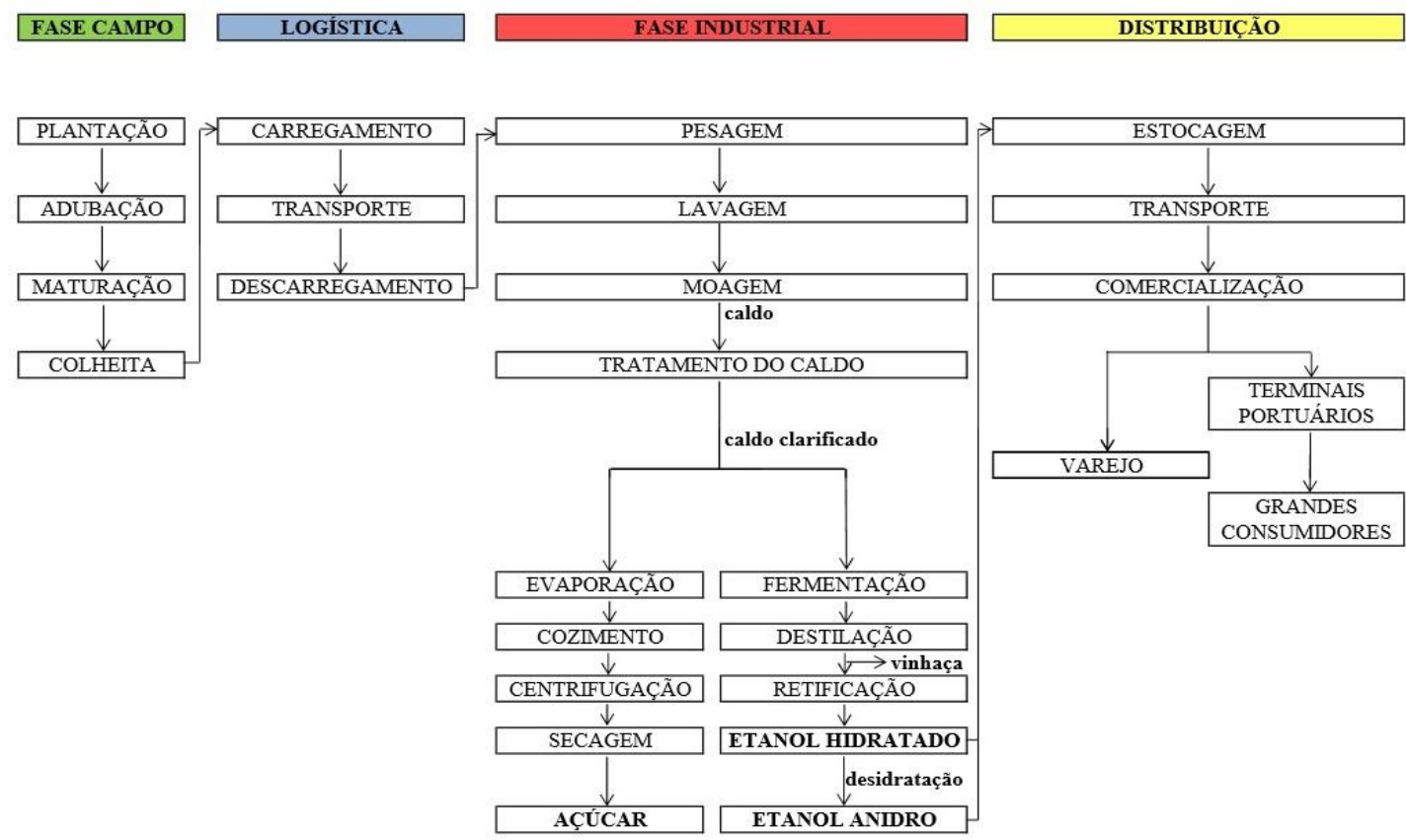

Figura 1. Cadeia Sucroenergética. Fonte: Lamarca et al., 2014.

\subsection{Etanol de Segunda Geração}

O bagaço da cana e a sobra de palha no campo tratam-se da biomassa resultante do etanol de primeira geração, que passou a ser importante não apenas para a produção de energia elétrica para as usinas, mas também para o aumento na produção de etanol. Desse modo, este processo é conhecido como etanol de segunda geração ou também como etanol celulósico (GALBE; ZACCHI, 2010). (Figura 2).
A produção do etanol de segunda geração requer um pré-tratamento, no qual as fibras do bagaço se abrem obtendo-se a polpa para passar pelo processo de hidrólise das enzimas, que fazem a quebra da lignina encontrada nos açúcares, de modo que ao ficarem livres inicia-se o processo de fermentação como acontece no processo de produção do etanol de primeira geração (BUCKERIDGE et a, 2010). 


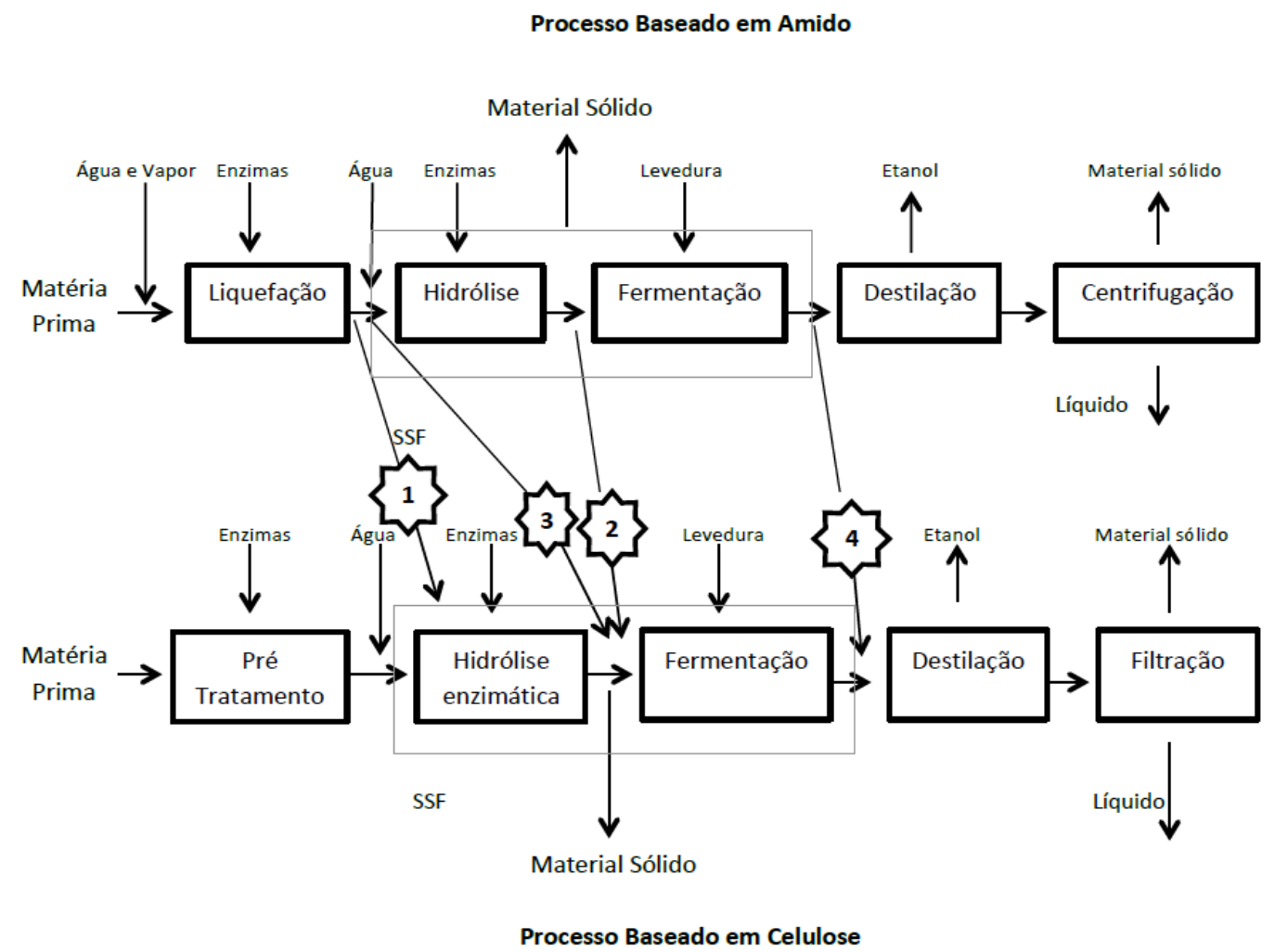

Figura 2. Fluxograma esquematizado dos possíveis pontos de integração entre produção de bioetanol de primeira e segunda geração. Fonte: Original de Galbe e Zacchi (2010;698).

De acordo com Buckeridge et al. (2010), este etanol possui as mesmas características do etanol oriundo da fermentação de sacarose, sendo assim, uma maneira de aumentar a sua produção na mesma área de plantio.

Segundo a União da Indústria de Cana de Açúcar (UNICA, 2014), o início da produção de etanol de segunda geração em escala comercial começou a ocorrer em setembro de 2014, na cidade de São Miguel dos Campos-AL, onde a empresa de biotecnologia Granbio possui

\section{PROCEDIMENTOS METODOLÓGICOS}

A produção de etanol a partir da biomassa começo começou a obter novas possibilidades e também a ganhar dinamismo no ano de 2008, com o apoio da Fundação de Amparo à Pesquisa do Estado de São Paulo (FAPESP), por meio do programa de Pesquisa em Bioenergia (BIOEN). Este programa, por sua vez, em Janeiro de 2015 estava formado por um conglomerado de 510 trabalhos divididos capacidade para produzir 82 milhões de litros por ano.

Os projetos que são analisados neste trabalho compõem-se das pesquisas do Programa BIOEN, envolvendo as tecnologias de produção de etanol de segunda geração, sendo que entre elas, estão: controle biológico, conversão enzimática, deslignificação, fixação de nitrogênio, genômica na fotossíntese, genótipos, hidrólise enzimática, marcadores moleculares e transcrição genética. entre auxílios à pesquisa e bolsas (FAPESP, 2015). Silva Junior e Silveira (2013) elaboraram critérios para uma préclassificação de tecnologias da produção de etanol de segunda geração, na qual são objetos de pesquisa dos projetos BIOEN, e desse modo entre as tecnologias consideradas como mais importantes neste segmento estão: hidrólise enzimática, marcadores moleculares, deslignificação, 
genótipos, conversão enzimática, transcrição genética, fixação de nitrogênio, genômica na fotossíntese e controle biológico.

Quadro 1. Principais temas de P\&D dos projetos BIOEN

\begin{tabular}{|l|c|}
\hline Temas de Pesquisa e Desenvolvimento & $\begin{array}{c}\text { Quantidade de Projetos, Auxílios e } \\
\text { Bolsas }\end{array}$ \\
\hline Hidrólise Enzimática & 105 \\
\hline Transcrição Genética & 91 \\
\hline Genótipos & 37 \\
\hline Conversão Enzimática & 29 \\
\hline Marcadores Moleculares & 21 \\
\hline Controle Biológico & 13 \\
\hline Genômica na Fotossíntese & 10 \\
\hline Fixação de Nitrogênio & 9 \\
\hline Deslignificação & 4 \\
\hline Total de Projetos, Auxílios e Bolsas & $\mathbf{3 1 9}$ \\
\hline
\end{tabular}

Fonte: Compilado a partir de dados da Biblioteca Virtual da Fapesp (2015).

Ao levar em consideração essas tecnologias de produção, foram classificados 319 projetos do BIOEN (Quadro 1) agrupando os trabalhos por tipos de tecnologias. É importante destacar que mais da metade das pesquisas estão concentradas nos temas de hidrólise enzimática (105) e transcrição genética (91). Esse evento deve ocorrer pelo fato de que a primeira trata de umas das principais barreiras para o desenvolvimento comercial de plantas produtivas em larga escala de etanol de segunda geração. Juntamente com esta ocorrência, temos a transcrição genética em que se refere a projetos sobre mecanismos de sinalização para ativação e repressão gênica, relacionados a processo de metabolismo de sacarose, relógio biológico, entre outros. Desse modo, trata-se de uma ferramenta básica para o desenvolvimento de ferramentas biotecnológicas específicas para a cana de açúcar.

Considerando a estrutura apresentada no quadro 1 , o presente estudo utilizou um modelo multicritério a partir do método de Análise Hierárquica de Processos (AHP), por se tratar de um problema discreto e pelo fato de ser um dos métodos mais utilizados dentro da escola americana (REIS et al, 2010).

Ao dividir os problemas em níveis hierárquicos, facilita a compreensão e avaliação do problema em estudo. A Figura 3 exibe o exemplo da metodologia hierárquica aplicada à análise do objetivo e também dos critérios, subcritérios e alternativas para aquisição de um automóvel. 


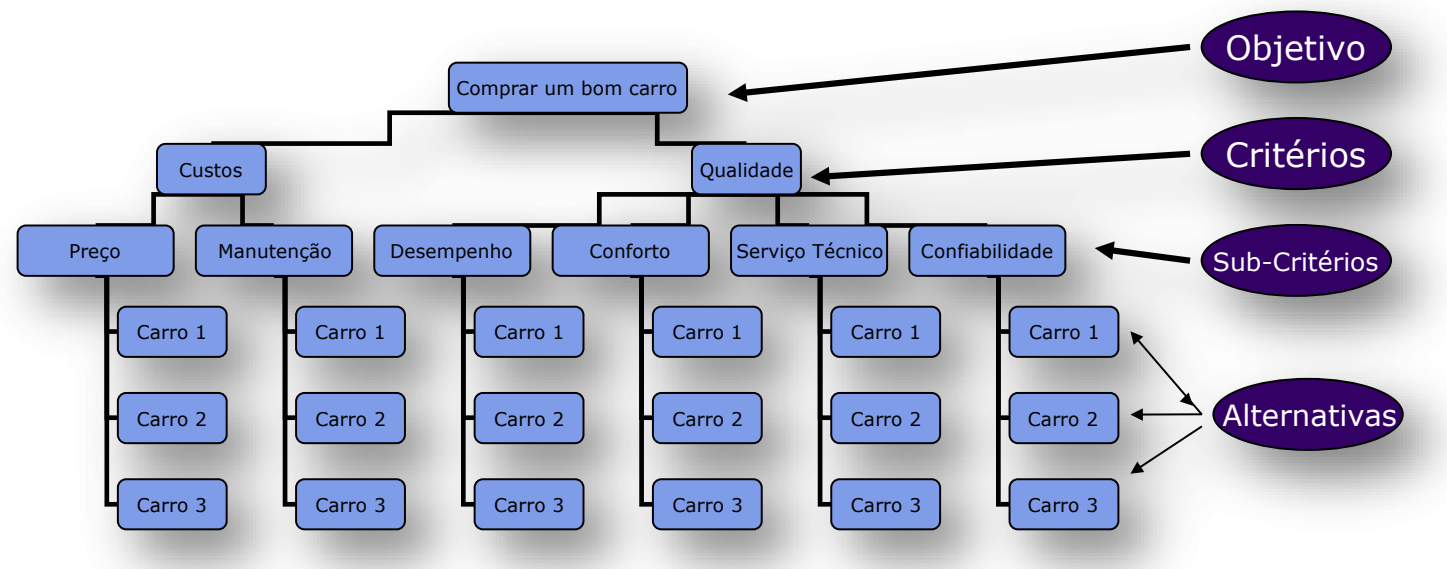

Figura 3. Hierarquia de objetivo, critérios, subcritérios e alternativas envolvidas na decisão para aquisição de um veículo. Fonte: Gomes et al., 2006.

A metodologia AHP, aplica-se em três etapas: (I) estruturação da hierarquia; (II) agregação das preferências dos especialistas e; (III) operacionalização da matriz de referência para obtenção dos vetores de prioridades (SAATY, 1991). Na primeira etapa, o problema é estruturado em uma árvore hierárquica desdobrando-se em critérios, subcritérios e alternativas (Figura 3). Na segunda, distribui-se a importância de cada variável da hierarquia de nível $n$, sobre a hierarquia $n+1$, onde o grau de importância é obtido por meio de uma comparação binária entre as variáveis, dentro de um dado nível hierárquico.

\footnotetext{
Entre os critérios apresentados a seguir - "Custos elevados da pesquisa ao produto final" e "Barreira comercial para produtos nacionais no mercado externo" - qual é mais importante em relação aos "Desafios econômicos"? Clique no gráfico para indicação da importância relativa entre os critérios.
}

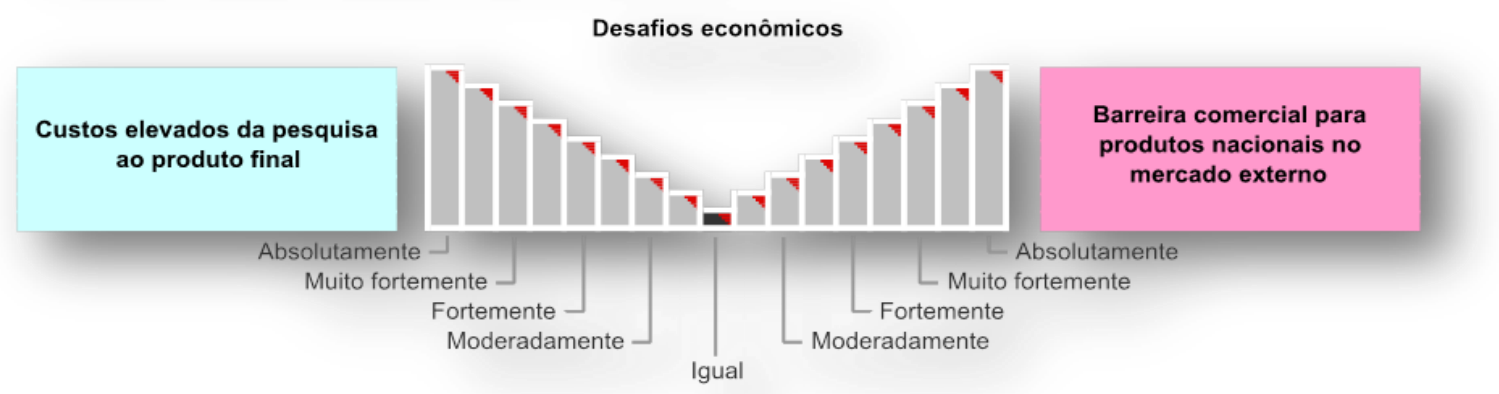

Figura 4. Comparação entre pares de critérios. Fonte: Pereira, A. M., 2009.

No Brasil, o exemplo de comparação binária em função de um objetivo usado no estudo de bioprospecção está representado pela Figura 4. Sendo que, a matriz utilizada nas comparações binárias está entre as variáveis de cada nível hierárquico, nos quais recebem a opinião de especialistas para avaliar seu grau de importância. A partir disso, normaliza-se a Matriz A, utilizando-se vetores de prioridade relativa e composta, representando assim, os pesos de cada variável na hierarquia de critérios, subcritérios e alternativas sob avaliação (SAATY, 1991). 
Quadro 2. Questionário elaborado para a pesquisa

\begin{tabular}{|l|}
\hline \multicolumn{1}{|c|}{ Questões que avaliaram o grau de importância } \\
\hline "Cultivares" e "Cultura" - qual é mais importante em relação à etapa "Agrícola" ? \\
\hline "Cultivares" e "Temas Específicos" - qual é mais importante em relação à etapa \\
"Agrícola" ? \\
\hline "Cultura" e "Temas Específicos" - qual é mais importante em relação à etapa "Agrícola" ? \\
\hline "Melhoramento Genético Tradicional" e "Melhoramento Genético Biotecnológico" - \\
qual é mais importante em relação à etapa "Cultivares" ? \\
\hline "Melhoramento Genético Tradicional" e "Marcadores Moleculares" - qual é mais \\
importante em relação à etapa "Cultivares" ? \\
\hline "Melhoramento Genético Biotecnológico" e "Marcadores Moleculares" - qual é mais \\
importante em relação à etapa "Cultivares" ? \\
\hline "Solo" e "Fertilizantes" - qual é mais importante em relação à etapa "Cultura" ? \\
\hline "Solo" e "Pragas e Toxicologia" - qual é mais importante em relação à etapa "Cultura" ? \\
\hline "Fertilizantes" e "Pragas e Toxicologia" - qual é mais importante em relação à etapa \\
"Cultura" ? \\
\hline "Tecnologias de Produção" e "Enzimas" - qual é mais importante em relação à etapa \\
"Técnicas e Insumos de Produção" ?
\end{tabular}

Sendo assim, depois de realizado um levantamento bibliográfico acerca do assunto relacionado aos processos produtivos de biocombustível e também da análise realizada no Quadro 1, na qual trata sobre os principais temas de P\&D dos projetos BIOEN, foi elaborada uma estrutura hierárquica (Figura 5) e as questões apresentadas no quadro 2 que permitiram separar em quatro níveis para agregação dos projetos de acordo com cada etapa do processo produtivo. Contudo, o objetivo deste trabalho é realizar uma análise apenas dos projetos que estão ligados à etapa agrícola da produção de etanol de segunda geração, ou seja, a estrutura hierárquica a seguir está composta com os temas ligados apenas a esta etapa. 


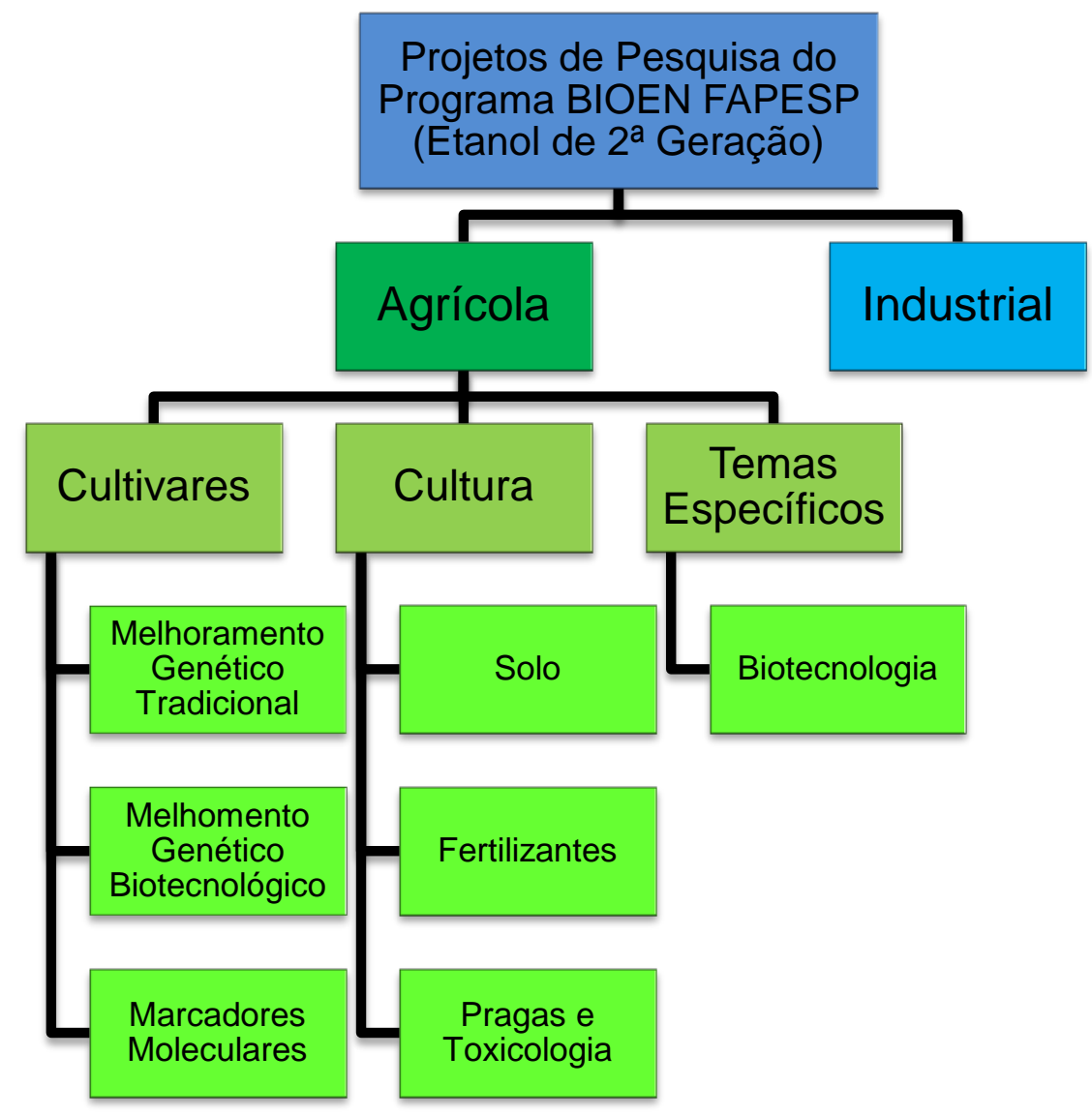

Figura 5. Estrutura Hierárquica dos Itinerários Tecnológicos para a produção de Biocombustível.

O questionário do quadro 2 foi enviado via correio eletrônico para especialistas de várias instituições que possuem relação direta com o setor sucroenergético. Entre essas instituições, estão: o Laboratório Nacional de Ciência e Tecnologia do Bioetanol (CTBE), Laboratório de Fisiologia Ecológica de

\section{ANÁLISE DOS RESULTADOS}

Assim sendo, após a elaboração e formulação do questionário, obteve-se respostas de 17 profissionais que atuam no setor sucroenergético, servindo como base para a construção das carteiras de projetos. Desse modo, os dados coletados a partir das entrevistas foram utilizados para preencher as Matrizes de Comparação e Normalização.

No questionário que foi enviado via correio eletrônico foi realizada uma comparação par a par entre os critérios analisados na Figura 5, com a finalidade de
Plantas (Lafieco) ligado ao Departamento de Botânica da Universidade de São Paulo (USP) e ao Núcleo de Apoio à Gestão da Inovação para a sustentabilidade no setor SucroEnergético (NAGISE) ligado a Universidade Estadual de Campinas, entre outras empresas que atuam no setor de bioenergia.

inserir as respostas obtidas nas Matrizes de Comparação de acordo com cada um dos níveis hierárquicos.

Essas matrizes por sua vez fazem parte do processo da metodologia de Análise Multicritério, na qual neste trabalho é inserida utilizando-se da Análise Hierárquica de Processos (AHP). A partir disso, na primeira etapa deste processo foi necessária a inserção das informações obtidas por meio do questionário, aplicando-se os dados na Matriz de Comparação de acordo com cada questão. 
$\mathrm{Na}$ segunda etapa, foi preciso inserir os dados obtidos na Matriz Normalizada a fim de padronizar as informações em uma mesma escala.

Após o preenchimento das Matrizes Normalizadas de cada um dos níveis, foi obtido o vetor de prioridade relativa, na qual é o resultado normalizado em uma escala padrão, da preferência dada por cada um dos especialistas. Sendo assim, foi feita uma média com as respostas dos entrevistados, pois leva em consideração ás respostas de todos especialistas, tendo como objetivo obter apenas um valor geral para cada item analisado. Nesse sentido, a Figura 6 ilustra o resultado de cada item analisado nos níveis da estrutura hierárquica, após a realização da tabulação das preferências manifestadas pelos especialistas.

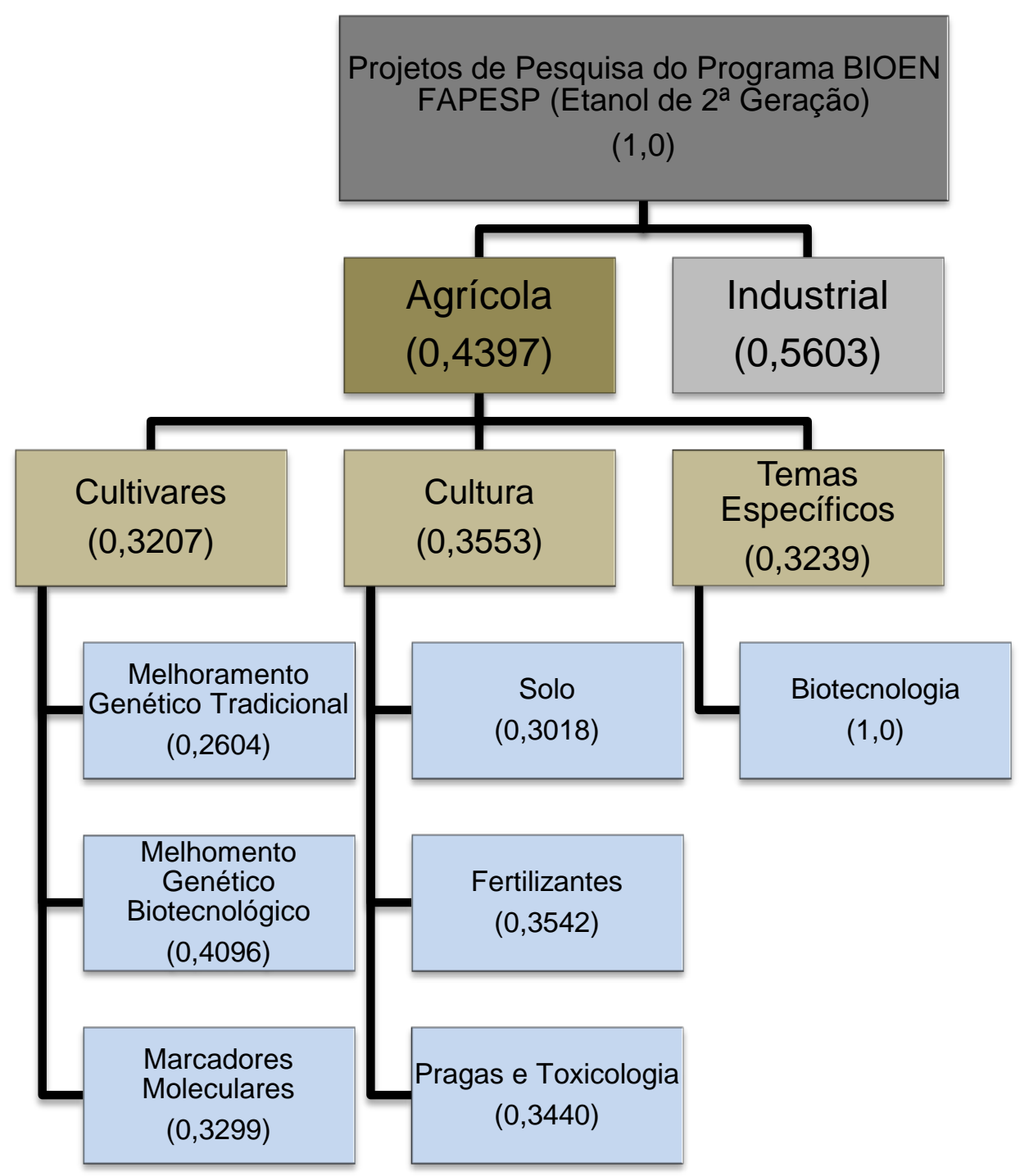

Figura 6. Estrutura Hierárquica dos Itinerários Tecnológicos para a produção de Biocombustível após Prioridade Relativa indicada por especialistas.

A obtenção dos vetores de prioridades relativas permitiu a identificação dos pesos dos critérios considerando apenas o valor dentro de cada elo da estrutura. Nesse contexto, foi preciso uma nova operação para realizar o cálculo dos vetores de prioridades compostas, na qual identifica os pesos de cada critério e subcritério levando em consideração todos os outros critérios da estrutura. A seguir, o Quadro 3 mostra o vetor de prioridade composta separado por 
cada um dos critérios e subcritérios analisados neste trabalho.

Quadro 3. Vetor de Prioridade Composta

\begin{tabular}{|l|c|}
\hline Critérios e Subcritérios & Vetor de Prioridade Composta \\
\hline $\begin{array}{l}\text { Projetos de Pesquisa BIOEN Etanol de } 2^{\text {a }} \text { geração } \\
\text { (Auxílios e Pesquisas). }\end{array}$ & 1,0 \\
\hline Agrícola & 0,4397 \\
\hline Cultivares & 0,1410 \\
\hline Melhoramento Genético Tradicional & 0,0367 \\
\hline Melhoramento Genético Biotecnológico & 0,0577 \\
\hline Marcadores Moleculares & 0,0465 \\
\hline Cultura & 0,1562 \\
\hline Solo & 0,0471 \\
\hline Fertilizantes & 0,0553 \\
\hline Pragas e Toxicologia & 0,0537 \\
\hline Temas Específicos & 0,1424 \\
\hline Biotecnologia & 0,1424 \\
\hline Industrial & 0,5603 \\
\hline
\end{tabular}

Fonte: Elaborado pelos autores a partir dos resultados obtidos na ilustrados na Figura 6.

No Quadro 3, percebeu-se que alguns itens se destacaram, obtendo um peso maior dentro do seu nível. No nível 2 da estrutura, identificou-se que ocorreu uma pequena diferença de 0,1206 entre as etapas Agrícola e Industrial. É importante destacar que os itens relacionados com a etapa industrial agregaram maior peso entre as preferências dos especialistas de maneira geral na produção de biocombustível.

Já no nível 3 da estrutura, foi subdividido em três etapas, sendo elas: Cultivares, Cultura e Temas Específicos. Dentre essas etapas, é importante destacar que de acordo com a preferência dada pelos especialistas do setor, a etapa Cultura foi a que obteve maior peso, seguido de Temas Específicos e Cultivares. Contudo, notou-se que a diferença de peso entre esses itens foi pouco significativa diante do contexto geral.

Após a realização de todas as etapas necessárias, na qual foi composta pela formação da estrutura hierárquica, atribuição dos pesos dos especialistas para cada nível da estrutura e o preenchimento dos vetores de prioridades relativa $\mathrm{e}$ compostas, foi possível o julgamento dos projetos realizando a seleção e organização desses projetos de P\&D do BIOEN em uma ordenação permitindo a formulação das carteiras de projetos.

Sendo assim, a partir dos resultados obtidos foram elaboradas cinco carteiras de projetos, divididas em: A, B, C, D e E. Essa classificação foi feita agrupando os projetos de acordo com as etapas em que estão inseridos no processo produtivo da cadeia sucroenergética. O portfólio das carteiras ficou separado de acordo com o Quadro 4.

A separação das carteiras em projetos foi realizada de acordo com as etapas produtivas ligadas à fase agrícola, na qual podemos analisar que a soma do número de projetos das carteiras $\mathrm{C}, \mathrm{D}$ e $\mathrm{E}$, representam a totalidade de projetos de P\&D ligados à etapa agrícola do programa BIOEN analisados neste trabalho. Nesse contexto, a carteira A representa todos os projetos da etapa agrícola e possuiu um peso de 0,4397, sendo que é o valor atribuído a esta fase quando comparado com a etapa industrial. 
Quadro 4. Classificação dos Projetos em Carteiras

\begin{tabular}{|c|c|c|c|}
\hline $\begin{array}{c}\text { Classificação } \\
\text { da Carteira }\end{array}$ & Composição da Carteira & $\begin{array}{c}\text { Vetor da } \\
\text { Prioridade } \\
\text { Composta da } \\
\text { Carteira }\end{array}$ & $\begin{array}{c}\text { Quantidade de } \\
\text { Projetos } \\
\text { localizados no } \\
\text { BIOEN }\end{array}$ \\
\hline A & Projetos da etapa Agrícola & 0,4397 & 181 \\
\hline B & $\begin{array}{c}\text { Projetos da etapa } \\
\text { Industrial }\end{array}$ & 0,5603 & 138 \\
\hline C & $\begin{array}{c}\text { Projetos da etapa } \\
\text { Cultivares }\end{array}$ & 0,1410 & 149 \\
\hline D & Projetos da etapa Cultura & 0,1562 & 10 \\
\hline E & $\begin{array}{c}\text { Projetos da etapa Temas } \\
\text { Específicos }\end{array}$ & 0,1424 & 22 \\
\hline
\end{tabular}

Desse modo, por um sistema de herança, os projetos que compõem as carteiras C, D e E são os mesmos contidos na carteira A. Sendo assim, ocorre a subdivisão nas carteiras C, D e Eque estão

\section{CONSIDERAÇÕES FINAIS}

Neste trabalho foram elaboradas cinco carteiras de projetos a partir de uma análise restrita de projetos do Programa de Pesquisa em Bioenergia (BIOEN), da Fundação de Amparo à Pesquisa do Estado de São Paulo (FAPESP), na qual se construiu a partir da utilização de metodologias de análise hierárquica de processos.

Quando desenhada a estrutura hierárquica utilizando o método AHP, obtém-se uma melhor divisão das áreas analisadas neste trabalho, possibilitando a distinção entre as etapas de fabricação do etanol e identificando a ligação com os processos de produção.

A divisão dos projetos em carteiras permitiu uma análise da classificação dos projetos ligados à etapa agrícola dentro do Programa em Bioenergia (BIOEN), realizando a divisão em agrupamentos fazendo com que a carteira A seja composta por todos os projetos ligados à etapa agrícola, a carteira B por todos os projetos ligados à etapa industrial $\mathrm{e}$ as carteiras C, D e E, por sua vez, compostas localizadas no quarto nível da estrutura hierárquica, pois procuram analisar de maneira mais específica as tecnologias dentro da etapa agrícola.

pelos projetos relacionados às etapas de Cultura, Cultivares e Temas Específicos, respectivamente.

Adjunto a esta divisão, houve o cálculo dos vetores de prioridades relativas e compostas, no qual a análise apenas do primeiro vetor não se mostrou suficiente para a análise e composição dos projetos em carteiras. Assim sendo, constatou-se a importância da obtenção dos vetores de propriedades compostas, gerando uma análise acerca de cada projeto em relação aos demais, de maneira a possibilitar a construção das carteiras.

Essa divisão em carteiras ocorreu para que se houvesse uma análise mais detalhada a respeito de cada etapa analisada. A carteira A representa todos os projetos da etapa agrícola e possuiu um peso de 0,4397, sendo que é o valor atribuído a esta fase quando comparado com etapa industrial, ou seja, essa carteira possui uma visão de modo mais geral de todo o processo ligado à etapa agrícola. A carteira B agrupou todos os projetos da fase industrial do processo. 
Sendo assim, foi possível perceber que entre as carteiras C, D e E, compostas por trabalhos ligados a Cultivares, Cultura e Temas Específicos, respectivamente, houve umamaior agregação de peso na carteira D (Cultura),mostrando sua maior importância em relação àsdemais carteiras C e E. Contudo, é importante destacar que a quantidade de projetos na carteira $\mathrm{C}$ se mostra muito superior em relação às carteiras D e E, significando que na prática

\section{REFERÊNCIAS BIBLIOGRÁFICAS}

ALONSO, Vera Lucia Chaves; JUNIOR, Nelson ALONSO; JUNIOR, Sergio Silva BRAGA. Inovação Tecnológica Denox: Uma Contribuição Para Mitigação Dos Gases De Efeito Estufa/Technological Innovation Denox: A Contribution For Mitigation Of Greenhouse Gas. Revista Brasileira de Engenharia de Biossistemas, v. 6, n. 3, p. 102-116, 2012.

BUCKERIDGE, M. S.; SANTOS, W. D.; SOUZA, A. P. As rotas para o etanol celulósico no Brasil. In: CORTEZ, L. A. B. (coord.) Bioetanol de cana-de-açúcar: P\&D para produtividade e sustentabilidade.São Paulo: Blucher, 2010. p.365-380.

COOPER, Martha C.; ELLRAM, Lisa M. Characteristics of supply chain management and the implications for purchasing and logistics strategy. The International Journal of Logistics Management, v. 4, n. 2, p. 13-24, 1993.

DeVELLIS, R. F. Scale Development: Theory and Applications. Thousand Oaks: Sage Publications, 2003.

FUNDAÇÃO DE AMPARO A PESQUISA DO ESTADO DE SÃO PAULO (FAPESP). Pesquisa em Bioenergia (BIOEN). São Paulo: FAPESP. 2014. Disponível em: http://www.bv.fapesp.br/pt/16/pesquisaem-bioenergia-bioen/. Acesso em: 22 nov 2014. ocorre uma quantidade muito alta de estudo envolvendo o tema Cultivares.

Nota-se então, que os resultados oriundos destes projetos auxiliaram para a avaliação dos impactos potencias do Programa BIOEN para o Estado de São Paulo e para a economia nacional, gerando-se informações para as tomadas de decisões para os planos de negócios, nos quais, estão dispostos na aplicação dos produtos e processos do BIOEN.

FUNDAÇÃO DE AMPARO A PESQUISA DO ESTADO DE SÃO PAULO (FAPESP). Pesquisa em Bioenergia (BIOEN). São Paulo: FAPESP. 2014. Disponível em: http://www.bv.fapesp.br/pt/16/pesquisaem-bioenergia-bioen/. Acesso em: 20 jan 2015.

GALBE, M.; ZACCHI, G. Produção de etanol a partir de materiais lignocelulósicos. In: CORTEZ, L. A. B. (coord.) Bioetanol de cana-de-açúcar: P\&D para produtividade e sustentabilidade. São Paulo: Blucher, 2010. pp.697-716.

GOMES, L. F. A. M.; GOMES, C. F. S.; ALMEIDA, A. T. Tomada de Decisão Gerencial: Enfoque Multicritério. São Paulo: Atlas, 2006.

LAMARCA, D. S. F.; BRAGA JUNIOR, S. S.; MAGALHÃES, M.M. Análise da Inserção dos Projetos de Pesquisa do BIOEN dentro da Cadeia Sucroenergética. Brasilian Journal of Biosystems Engineering. v 8(3). p. 234-249, 2014.

MIHM, J.; LOCH, C.; HUCHZERMEIER, A.Problem-solving oscillations in complex engineering projects. Management Science, Providence, v.46, n.6, p.733-750, June 2003. 
PEREIRA, A. M. Condicionantes institucionais para bioprospecção no Brasil. Campinas, 2009. 290f. Dissertação (Mestrado em Economia) Instituto de Economia, Universidade Estadual de Campinas.

REIS, J.G.M; NÄÄS, I.A; COSTA NETO, P.L.O.; VENDRAMETTO, O. Impacto Da Qualidade Do Produto Na Seleção Do Mercado De Carne Bovina Utilizando Análise Multicriterial/Impact Of The Product Quality On The Selection Of Beef Market Using Multicriterial Analysis. Revista Brasileira de Engenharia de Biossistemas, v. 4, n. 1, p. 24-39, 2010.

RENEWABLE FUELS ASSOCIATION. GOING GLOBAL. Washington, DC: RFA, 2015. 36 p. (2015 Ethanol Industry
Outlook). Disponível em: $<$ http://ethanolrfa.3cdn.net/c5088b8e8e6b4 27bb3_cwm626ws2.pdf>. Acesso em: 04 mai 2015.

SAATY, T. L. Método de Análise Hierárquica. São Paulo: McGraw-Hill, Makron, 1991.

SILVA JUNIOR, J. J.; SILVEIRA, C. B. Pesquisa básica e progresso tecnológico na indústria brasileira de etanol de biomassa de cana-de-açúcar. Relatório de pesquisa. (mimeo). 2013. 16p.

UNIÃO DA INDÚSTRIA DE CANA DE AÇÚCAR - UNICA. Indústria Brasileira de Cana-de-Açúcar.Uma Trajetória de Evolução. Disponível em: <http://www.unica.com.br/linhadotempo/i ndex.html> Acesso em: 20 out 2014. 